\title{
GrubiBots Educacional: jogo para o ensino de algoritmos na educação básica
}

\author{
Gabriela A. A. de Oliveira ${ }^{1}$, Raphael W. de Bettio ${ }^{2}$, Ana P. M. Rodarte ${ }^{3}$, Jussara E. \\ Braz $^{3}$, Fernanda B. Ferrari ${ }^{3}$
}

12 Departamento de Ciência da Computação - Universidade Federal de Lavras (UFLA) Caixa Postal 3037 - 37200-000 - Lavras - MG - Brasil

${ }^{3}$ Departamento de Educação - Universidade Federal de Lavras (UFLA) - Caixa Postal 3037 - 37200-000 - Lavras - MG - Brasil

\{goliveira, raphaelwb\}@dcc.ufla.br,
anapaula_menezesrodarte@yahoo.com.br, brazeliz33@yahoo.com.br, feferrari@ded.ufla.br

Abstract. This work has as main objective the presentation of the game called Educational GrubiBots, this game is a program for teaching algorithms in early childhood education with the support of robotic tool. The game was developed with the aim of contribute to the current scenario of educational games aimed at children, since the application of computing as a basic science is still considered novice in Brazil.

Resumo. O presente trabalho possui como objetivo principal a apresentação do jogo denominado GrubiBots Educacional, este jogo é uma aplicação para $o$ ensino de algoritmos na educação infantil com o apoio de ferramenta robótica. $O$ jogo foi desenvolvido com o intuito de contribuir para o atual cenário de jogos educacionais voltados para o público infantil, uma vez que a aplicação da computação como uma ciência básica ainda é considerada principiante no Brasil.

\section{Introdução}

A Sociedade Brasileira de Computação (SBC) tem como um dos seus objetivos institucionais incentivar atividades de ensino, pesquisa e desenvolvimento em computação no Brasil. Assim, a SBC admite que os princípios e habilidades introdutórias de computação devem ser desenvolvidos pelos estudantes desde os primórdios da educação básica. Segundo Wing (2006), esse subconjunto de competências e habilidades pode ser denominado como pensamento computacional, envolvendo as competências relacionadas à abstração e decomposição de problemas de forma a permitir sua resolução usando recursos computacionais e estratégias algorítmicas.

O CSTA K-12 Computer Science Standards ressalta a necessidade de desenvolvimento de habilidades computacionais na Educação Básica, auxiliando no desenvolvimento de capacidades de resolução de problemas, dando suporte e se relacionando com outras ciências, além de atuar como um elemento motivador para os estudantes. Ainda segundo CSTA, representar a solução para uma determinada situação na linguagem algorítmica é uma das competências fundamentais do pensamento computacional (CSTA, 2011).

Atualmente, o ensino de algoritmos no Brasil se restringe à educação superior ou pós-graduação. A aplicação da computação como uma ciência básica é ainda incipiente quando contexto é a educação básica brasileira. A partir do panorama exposto, é evidente a importância de viabilizar o conhecimento tecnológico-científico e estimular o desenvolvimento do pensamento lógico pelos estudantes de ensino básico. Soma-se a este quadro a experimentação de tecnologias educacionais com forte apelo lúdico, o que 
se torna propício através da robótica educacional.

De acordo com Zilli (2004), a robótica educacional potencializa as seguintes capacidades: raciocínio lógico; habilidades manuais e estéticas; relações interpessoais e intrapessoais; emprego de conceitos adquiridos em diferentes áreas do conhecimento para o desenvolvimento de projetos; investigação e compreensão; representação e comunicação; trabalho com pesquisa; resolução de problemas por meio de erros e acertos; aplicação das teorias formuladas a atividades concretas; utilização da criatividade em diferentes situações; capacidade crítica.

Nesse contexto, a robótica têm se apresentado como um domínio para o ensino de programação para crianças. A robótica educacional pode ser utilizada para motivar e despertar o interesse dos alunos em disciplinas muitas vezes consideradas de difícil entendimento, como, por exemplo, as de programação, devido ao alto índice de abstração exigido [Benitti et al 2009].

Diante disso, essa pesquisa propõe o desenvolvimento de um jogo educacional para o ensino de algoritmos na educação básica, o qual possui como ferramenta de apoio a robótica. O jogo possui como diretrizes principais o desenvolvimento de habilidades lógicas computacionais pelos alunos e a difusão do ensino de algoritmos de forma lúdica. Conforme (Melo, 2013), os softwares na área de Ciência da Computação são, de modo geral, voltados ao ensino superior e têm como objetivo incentivar ou auxiliar a fixação de conteúdos desse nível de ensino, logo o desenvolvimento de jogos digitais com apelo ao lúdico que aborde os conceitos inerentes à grande área da Ciência da Computação apresenta-se com um campo de investigação desafiador para alunos licenciandos em Ciências da Computação.

O presente artigo possui como objetivo principal apresentar o jogo, denominado GrubiBots Educacional, desenvolvido em conjunto pelo Departamento de Ciência da Computação e pelo Departamento de Educação da Universidade Federal de Lavras. Esse jogo tem o intuito de resolver problemas reais das diversas áreas do conhecimento, através do desenvolvimento de modelos algorítmicos aplicados à robótica.

O artigo está estruturado da seguinte forma: a seção 1 traz uma contextualização. A seção 2, nomeada de "Trabalhos Relacionados", apresenta uma série de jogos educativos existentes para o ensino de algoritmos e programação. Em sequência, a seção 3, denominada "Abordagem do GrubiBots Educacional" e considerada a principal seção, descreve de forma detalhada o jogo. A seção 4 apresenta as conclusões e os trabalhos futuros, vislumbrados a partir dos resultados já obtidos a partir do desenvolvimento do jogo.

\section{Trabalhos Relacionados}

Os jogos vêm ganhando espaço como ferramenta de ensino e aprendizagem, pode-se constatar que eles são um fator de grande contribuição para o desenvolvimento de habilidades cognitivas e motoras de indivíduos. Atualmente, os jogos digitais para o ensino da computação se diversificam entre aplicações para o ensino de algoritmos e estruturas de dados, assim como disciplinas mais específicas como Inteligência Artificial, Engenharia de Software, Interface Humano-Computador e Tecnologias para Web.

Dentro do domínio de jogos para o ensino de algoritmos e programação para crianças e adolescentes são reportados alguns trabalhos como o Camp CyberGirls (Hulsey, 2014), o qual explorou a ferramenta de introdução à programação conhecida como Curiosity Grid, esta baseia-se no desenvolvimento de código real para resolução de problemas de programação. O software Alice, reportado no trabalho de (G Morati Jr, 2012), tem como propósito apoiar estudantes na aprendizagem de programação orientada a objetos por meio da criação de animações e jogos em ambientes 3D. Outro trabalho da área aborda o jogo ProGrame, este software disponibiliza algoritmos pré- 
definidos para serem criados ou receberem manutenção em uma linguagem de programação a ser definida pelo professor. O trabalho de (Fessakis, 2012) aborda dois ambientes de desenvolvimento, o Ladybug leaf e o Ladybug maze, ambos baseados em Logo e permitem aos usuários utilizar comandos específicos para controlar a tartaruga Logo ou a joaninha até alcançar a folha ou resolver problemas de labirinto. O Scratch, descrito no trabalho de (Resnick, 2009), é um projeto de aprendizagem que permite o desenvolvimento de habilidades de programação na infância, através de um ambiente com design simples e atraente.

Considerando-se a programação de computadores, ainda encontram-se na literatura tecnologias educacionais para o ensino de algoritmos em que a ferramenta de apoio utilizada é a robótica. Um trabalho da área, desenvolvido por (D'Abreu, 2011), é baseado no uso de ambiente de Robótica com Br-Gogo, este software possui uma plataforma que permite a construção de programas para controlar o comportamento de robô físico, por meio de blocos gráficos. O trabalho de (Friedrich, 2012) propõe o ensino de lógica de programação com Logo e Lego Mindstorms. No trabalho desenvolvido por (Barokava, 2011) foi explorada a ferramenta Admoveo robot, a qual explora o uso de algoritmos de aprendizagem para treinar robôs com comandos de voz.

Nessa perspectiva, destacam-se o software denominado Robotimov (Dantas, 2013), este propôs o desenvolvimento de um parque temático onde robôs se encontram em diferentes situações cotidianas. $O$ jogo consiste de várias fases com desafios onde o jogador necessita desenvolver códigos na linguagem de programação Python para resolvê-los. Outro software a ser abordado é o RoboMind (Bentini, 2009), o qual é uma IDE (Integrated Development Environment) que possui uma linguagem de programação própria para criar situações de movimentação de um robô em um espaço bidimensional. Soma-se a este quadro a ferramenta que integra o Scribbler Robot e Alice, descrita no trabalho de (Salcedo, 2011). Alice é uma interface de desenvolvimento de programas que permite arrastar itens de um painel para outro para gerar código de programação em geral. Já o Scribbler é um robô totalmente programável, o qual possui vários sensores que permitem a interação do robô com o ambiente a sua volta.

Pode-se constatar que a maioria dos jogos digitais abordando o ensino de algoritmos são aplicados a disciplinas relacionadas à programação em cursos de graduação, considerando-se que os alunos apresentam alto nível de dificuldade para desenvolverem o raciocínio lógico necessário em tais disciplinas. Logo, os softwares nesta área com forte apelo lúdico apresentam-se como um campo desafiador e pouco explorado, principalmente, quando o cenário é o ensino infantil. Neste sentido, o presente artigo fundamenta-se em contribuir para o cenário de jogos digitais aplicados ao ensino de algoritmos para o público infantil.

\section{Abordagem do GrubiBots Educacional}

GrubiBots Educacional é um jogo resultante do desenvolvimento de projeto de pesquisa na área de Robótica Pedagógica. O que se pretende com a Robótica Pedagógica é expandir os métodos de ensino em computação voltados para a educação básica, com o enriquecimento e a diversificação das formas de se obter conhecimento.

\subsection{Descrição do GrubiBots Educacional}

O GrubiBots Educacional é uma plataforma de desenvolvimento que apresenta um método de programação inovador em relação aos tradicionais métodos de ensino e aprendizagem desta área, uma vez que, tal ferramenta possibilita a construção de algoritmos a partir da interconexão entre blocos de programação formados por instruções e estruturas de programação representadas por ícones, conforme ilustra a (Figura 1 (a)), que representa a área de desenvolvimento do programa.

Pode-se observar através da mesma figura que a interface é composta por duas 
áreas, à esquerda encontra-se o painel de controle da ferramenta, onde são apresentadas funcionalidades do jogo. À direita, é exibida a área de desenvolvimento dos algoritmos, os quais são compostos por blocos que atribuem comandos ao robô. Estes blocos podem ser arrastados para formar novos programas ou serem jogados ao lixo, através da lixeira exibida.

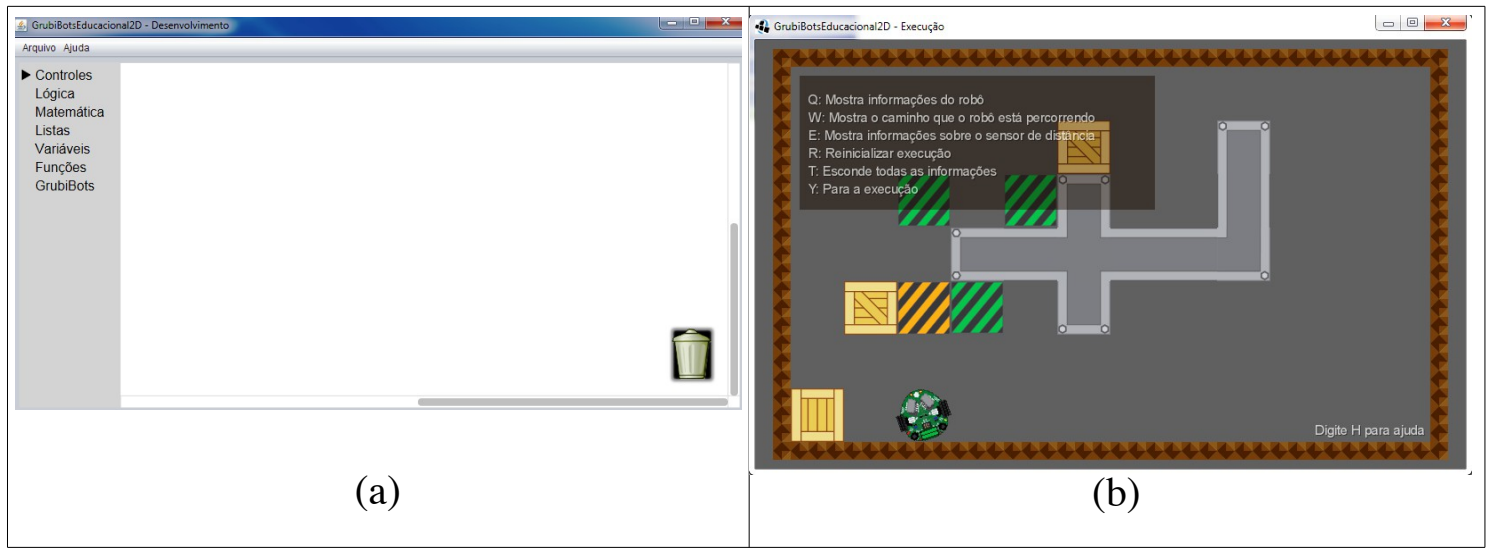

Figura 1 (a). Ambiente de Desenvolvimento, (b). Ambiente de Execução

Além do ambiente de desenvolvimento, o jogo possui uma área exclusiva para execução do robô (Figura 1 (b)). Observa-se que a execução ocorre em um espaço bidimensional formado por alguns componentes, como os blocos listrados que indicam início e fim de trajeto, caixas e áreas fechadas, as quais representam obstáculos do jogo. Este ambiente, também é composto pela opção denominada Digite H para ajuda, caso a letra $\mathrm{H}$ seja selecionada, tornam-se disponíveis uma série de alternativas que podem ser utilizadas para obter informações adicionais sobre a execução do jogo, tais alternativas também são exibidas na (Figura 1 (b)).

Em relação ao ambiente de execução, considera-se que a interface gráfica do jogo é totalmente maleável, uma vez que o jogo oferece opção para configurar o ambiente (Figura 2), ou seja, o ambiente do jogo é adaptável a aplicação do usuário, o que amplia a gama de atividades que podem ser resolvidas com o apoio da ferramenta GrubiBots Educacional. A configuração do ambiente é feita com base em conceitos de matriz, em que cada número observado nos campos brancos da (Figura 2), consiste em uma célula da matriz. Os blocos numerados de 1 até 14 são utilizados para formar caminhos ou obstáculos durante o jogo, já os blocos listrados em verde e laranja delimitam, respectivamente, o início e fim do trajeto.

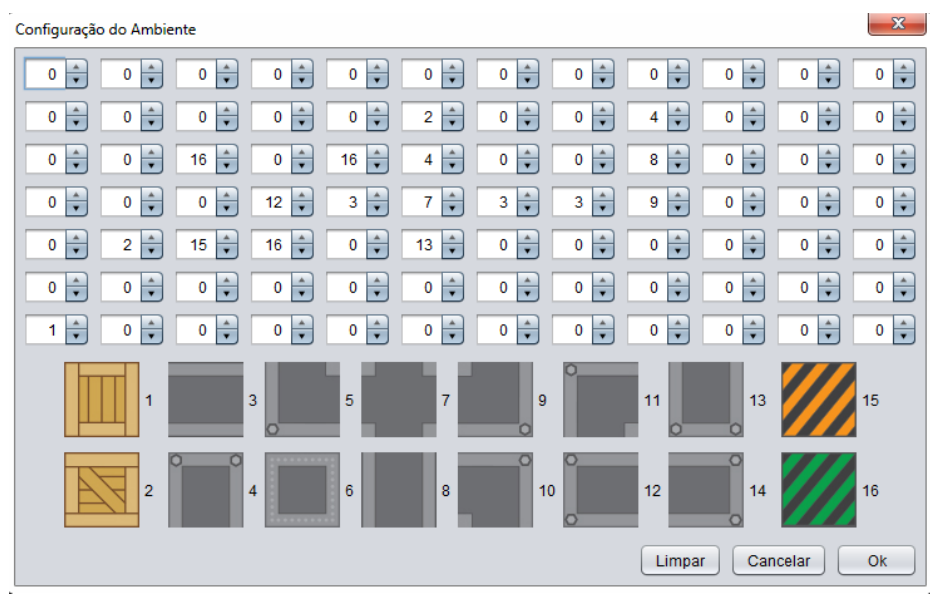

Figura 2. Tela de Configuração do Ambiente

Durante o desenvolvimento dos algoritmos, o jogo permite trabalhar com os 
blocos de instruções de forma rápida e simples, uma vez que ele oferece opções de edição de bloco que facilitam a construção dos algoritmos. Estas opções consistem em duplicar o bloco, adicionar/remover comentário, entrada interna/externa, recolher/expandir bloco, habilitar/desabilitar bloco, remover todos os blocos e ajuda.

A sintaxe da linguagem contida nos blocos de instruções é caracterizada por ser escrita em português e ser composta pelos métodos mais comuns de programação, como estruturas nomeadas para- $>f a c ̧ a$ e $s e->f a c ̧ a$, assim, os algoritmos podem ser criados independente do desenvolvimento de código em determinada linguagem de programação. Dentro deste domínio, ressalta-se que o jogo não causa erros de programação, o que evita a frustração por parte dos alunos e ainda contribui para fomentar o aprendizado de programação.

Dentre as características do GrubiBots que viabilizam a aplicação do jogo em escolas, destaca-se que o jogo é disponível gratuitamente em versão português, é possível utilizar a aplicação em computadores de baixo desempenho, além disso, o jogo possui grande portabilidade, uma vez que a aplicação é disponível tanto para o sistemas operacionais Windows, quanto para sistemas Linux e Mac. Estes fatores são considerados primordiais e decisivos para fazer do jogo uma estratégia de ensino para estudantes e promover a imersão do jogo em escolas, sem a geração de custo financeiro e contribuindo para a disseminação de conceitos de computação no ensino infantil.

\subsection{Plano de Ensino}

De modo a estimular o desenvolvimento de diferentes níveis cognitivos no aprendiz de programação, foi proposto o plano de ensino a seguir, abordando desde conceitos básicos que compõem o jogo até funcionalidades mais complexas.

O procedimento do jogo GrubiBots, tendo por intuito a inserção à lógica de programação para crianças, foi distribuído em objetivos específicos dentro dos temas abordados a seguir.

\subsubsection{Criação de variáveis}

Um conceito primordial para iniciar o desenvolvimento de um algoritmo é a definição de variável, esta é utilizada quando se deseja armazenar um valor. No jogo, variáveis podem ser criadas por meio da instrução denominada DEFINIR ITEM PARA, onde item é o nome que representa a variável e o mesmo pode ser alterado para definir novas variáveis. Cada variável declarada fica armazenada no painel de controle do jogo e disponível para utilização em diferentes atividades. Considerando-se que variáveis são utilizadas demasiadamente em atividades de programação, exemplos da utilização de variáveis serão apresentados nos próximos tópicos.

\subsubsection{Operadores lógicos e matemáticos}

Outra instrução muito utilizada em linguagem de programação são os operadores. Em programação, operadores são apresentados de acordo com dois tipos, operadores lógicos e operadores matemáticos. Assim como nas diversas áreas no conhecimento, os operadores lógicos são base para a construção de estruturas proposicionais, sendo os principais operadores lógicos representados em português por E, IGUAL, MAIOR, MENOR e suas derivações, os quais são operadores binários, ou seja, necessitam de dois elementos, e $\mathrm{NÂ} O$, um operador unário. Além desses operadores, o jogo disponibiliza as instruções lógicas que permitem atribuir uma variável ou sentença como verdadeiro/falso, nulo e realizar teste de veracidade.

Os operadores matemáticos oferecidos no jogo permitem realizar as quatro operações básicas de matemática - soma, subtração, multiplicação e divisão - como também operações logarítmicas; exponenciação; raiz quadrada; valor absoluto, positivo, negativo, nulo, par, ímpar, primo ou inteiro; fração aleatória, determinar se um número é divisível por outro e especificar um intervalo de valores para uma variável. Esta gama 
de operadores amplia o campo de atividades que podem ser resolvidas através do jogo. As aplicações do uso de operadores lógicos e matemáticos estarão presentes em atividades apresentadas nos temas a seguir.

\subsubsection{Estruturas de controle de repetição}

A linguagem do GrubiBots possui as estruturas de repetição REPITA e CONTAR como derivações das estruturas de programação for e while, respectivamente. Dentre estas estruturas, o jogo ainda apresenta opções diversas, como repetir determinado número de vezes uma sentença (REPITA $\mathrm{N}$ VEZES, FAÇA); repetir um bloco de instruções enquanto uma condição for verdadeira (REPITA ENQUANTO N, FAÇA); além de opções para contar um conjunto de instruções e executá-las, de acordo um intervalo com valor inicial, final e um incremento; contar um conjunto de itens em uma lista e executar instruções; ou encerrar determinado laço de repetição.

Um exemplo de algoritmo com laço de repetição é exibido na (Figura 3 (a)). Este programa atribui comandos para o robô andar 120 unidades e girar para direita $90^{\circ}$ enquanto a condição, numero1 < 5, é verdadeira, sendo que numero1 é uma variável inteira com valor inicial 0 . A cada execução do bloco de instruções contido na estrutura repita, a variável numero1 é incrementada em 1. O fim do laço se dá quando numero1 possui valor 5, assim o robô repete o laço 4 vezes e obtém-se como resultado a formação da figura geométrica plana quadrado, sendo que um lado foi executado duas vezes.

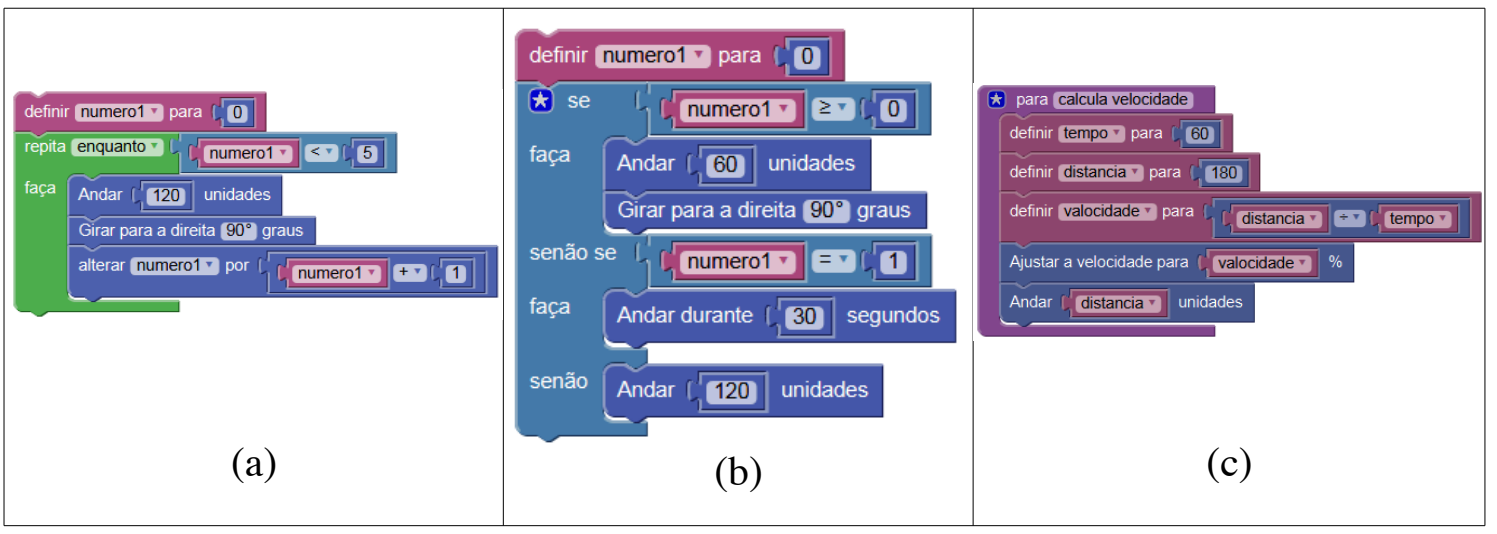

Figura 3 (a). Exemplo da estrutura de repetição, (b). Exemplo da estrutura condicional, (c). Exemplo de função

\subsubsection{Estruturas de controle condicional}

As estruturas condicionais do jogo consistem em derivações da estrutura if para SE. São oferecidas as estruturas (SE, FAÇA), (SE, FAÇA, SENÃO), (SE, FAÇA, SENÃOSE, FAÇA, SENÃO), as quais baseiam-se na mesma interpretação. Estas estruturas executam um sentença de instruções se determinada condição for satisfeita, ou seja, cada SE verifica se uma condição é verdadeira, em caso positivo, executa as instruções passadas dentro do bloco FAÇA, caso contrário, encerra a execução do laço.

A (Figura 3 (b)) apresenta um exemplo de algoritmo abordando estruturas de repetição, onde é verificada a condição, numero1 $>=0$, se esta for verdadeira, o robô anda 60 unidades e gira para direita 90 graus, caso a condição seja falsa, é testado se numero1 $=1$. Neste exemplo, a variável numero1 é definida com valor 0 , logo o primeiro bloco de instruções FAÇA é o único executado pelo robô.

Pode-se perceber que o jogo permite a criação de algoritmos adaptáveis a necessidades do usuário, além de explorar instruções simples até quando o algoritmo possui uma estrutura extensa.

\subsubsection{Criação de funções}


Dentre as funcionalidades do GrubiBots, as funções são consideradas de grande valia, elas permitem criar algoritmos que podem ser reutilizados em novos programas do jogo apenas com a utilização do bloco de instrução com o nome da função que se torna disponível no painel de controle do jogo. Esta funcionalidade torna o desenvolvimento de algoritmos mais rápido e contribui para o melhor desempenho do usuário durante o jogo.

A (Figura 3 (c)) apresenta um exemplo básico da aplicação de funções. O algoritmo representa a função calcula velocidade que é feita com base nos valores de tempo e distância definidos como variáveis da função. Após calcular a velocidade resultante, o algoritmo altera a velocidade do robô para o valor calculado e atribui o comando andar para a distância definida.

\subsubsection{Criação de listas}

O jogo também permite trabalhar com listas de programação, esta opção apresenta uma série de funcionalidades que otimizam o desenvolvimento de listas, como por exemplo, criar lista com um item repetido determinado número de vezes; encontrar na lista a ocorrência de um item em determinada posição, além de alternativas mais simples como criar lista vazia; criar lista de acordo com 3 tipos de sentenças diferentes, definir ou obter valor de um item em específico da lista. As listas são definidas para atuarem combinadas com estruturas de controle, pois seus itens são trabalhados por meio de instruções com verificações condicionais ou de repetição.

\subsubsection{Explorando o GrubiBots}

$\mathrm{O}$ robô possui um conjunto de instruções referentes aos seus atuadores. Ele pode movimentar para frente (ANDAR $\mathrm{N}$ UNIDADES). Além disso, o robô pode movimentar-se andando durante determinado tempo (ANDAR DURANTE $\mathrm{N}$ SEGUNDOS). Outra possibilidade de movimentação do robô é alterar sua velocidade (AJUSTA VELOCIDADE PARA N \%). O robô também pode virar-se o valor em graus desejado através da opção (GIRAR DIREITA/ESQUERDA N GRAUS) e virar-se durante determinado tempo, (GIRAR PARA DIREITA/ESQUEDA DURANTE $\mathrm{N}$ SEGUNDOS).

As aplicações de comandos específicos do GrubiBots é comum dentre todos os algoritmos desenvolvidos através deste jogo, são estes comandos que provêm o real movimento do robô. Exemplos da utilização de comandos andar e girar foram apresentados em tópicos anteriores deste plano de ensino.

O objetivo do jogo consiste em desenvolver algoritmos por meio interligação de blocos com instruções ou estruturas de programação dentro do domínio de objetivos apresentados, combinadas de acordo com o tipo de atividade a ser programada, o raciocínio lógico exigido pelo problema, e acima de tudo, deixando o usuário livre para montar seu algoritmo seguindo suas preferências.

\section{Conclusões e Trabalhos Futuros}

O desenvolvimento do jogo GrubiBots contribui para a área de jogos digitais aplicados ao ensino de lógica de programação de forma lúdica voltados, especialmente, para a disseminação de conceitos algorítmicos ainda na educação infantil com o intuito de introduzir a Ciência da Computação na educação básica. No tocante aos princípios propostos com o desenvolvimento do jogo, destacam-se os objetivos de fomentar o aprendizado de lógica de programação em crianças, promover o raciocínio lógico e o desenvolvimento de habilidades cognitivas em crianças. Além disso, pretende-se motivar os profissionais, principalmente da área de Ciência da Computação, a buscarem por novas propostas neste campo de atuação.

Embora os jogos educacionais descritos na seção de Trabalhos Relacionados proponham a imersão do aluno em seus ambientes, é evidente que o campo de jogos 
digitais lúdicos voltados para o público infantil ainda é considerado um domínio desafiador, principalmente, para profissionais da área de Ciência da Computação. Dentre as ferramentas descritas neste artigo, somente os trabalhos de (Fessakis, 2012), abordando os ambientes de desenvolvimento Ladybug leaf e o Ladybug maze e, o trabalho de (Friedrich, 2012), desenvolvido com Logo e Lego Mindstorms, são experimentações aplicadas ao público infantil. Em relação às demais ferramentas, apenas três delas, as quais são denominadas Robomind, CyberGirls e ambiente de robótica com Br-Gogo são aplicações voltadas para o ensino médio, todas as demais ferramentas são propostas para o ensino superior.

Fazendo uma analogia do jogo GrubiBots Educacional com as demais ferramentas voltadas para o público infantil, destaca-se que o GrubiBots é um jogo que não necessita do desenvolvimento de códigos de programação. Além disso, o jogo pode ser utilizado para resolver problemas de programação de diversas áreas de conhecimento, possui boa maleabilidade e fácil usabilidade.

Este artigo teve como objetivo principal descrever a ferramenta GrubiBots Educacional, com ênfase em suas funcionalidades. Publicações futuras serão destinadas a apresentação dos resultados obtidos a partir da aplicação da ferramenta para alunos de ensino básico. Além disso, está em desenvolvimento um portal educacional destinado a divulgação de materiais didáticos, roteiros de aula e da própria ferramenta, a qual se encontrada em estado de produção. Interessados no trabalho podem entrar em contato com os autores e serão informados quando o material estiver disponível.

\section{Agradecimentos}

Os autores agradecem à Coordenação de Aperfeiçoamento de Pessoal de Nível Superior (CAPES) e à Fundação de Amparo à Pesquisa do Estado de Minas Gerais (FAPEMIG) pelo auxílio financeiro fornecido para a realização desse trabalho. Também é feito um agradecimento à Prefeitura Municipal de Lavras pelo apoio prestado ao projeto. Destacam que o uso das seguintes bibliotecas de software foram imprescindíveis para o desenvolvimento: APACHE COMMONS, BLOCKLY, JACKSON e LIBGDX.

\section{Referências}

Aureliano, Viviane Cristina Oliveira, and Patrícia Cabral de Azevedo Restelli Tedesco. (2012) "Ensino-aprendizagem de Programação para Iniciantes: uma Revisão Sistemática da Literatura focada no SBIE e WIE." Anais do Simpósio Brasileiro de Informática na Educação. Vol. 23. No. 1.

Barakova, Emilia, Ruud Mestrom, and Willem Willemsen. (2011) "The application of learning algorithms in the development of natural interaction." Procedings of the Second Conference on Creativity and Innovation in Design. ACM.

Barbosa, Weider Alves, and Paulo Afonso Parreira Júnior. (2013) "Um Mapeamento Sistemático sobre Ferramentas de Apoio ao Ensino de Algoritmo e Estruturas de Dados." Anais do Simpósio Brasileiro de Informática na Educação. Vol. 24. No. 1.

Barcelos, T., and Ismar Frango Silveira. (2012) "Pensamento Computacional e Educação Matemática: Relações para o Ensino de Computação na Educação Básica." XX Workshop sobre Educação em Computação, Curitiba. Anais do XXXII CSBC.

Benitti, Fabiane Barreto Vavassori, et al. (2009) "Experimentação com Robótica Educativa no Ensino Médio: ambiente, atividades e resultados." Anais do Workshop de Informática na Escola. Vol. 1. No. 1.

D'Abreu, João Vilhete V., Luiz GB Mirisola, and Josué JG Ramos. (2011) "Ambiente de Robótica Pedagógica com Br_GOGO e Computadores de Baixo Custo: Uma Contribuição para o Ensino Médio." Anais do Simpósio Brasileiro de Informática na Educação. Vol. 1. No. 1.

Dantas, Vanessa F., et al. (2013) "Combinando desafios e aventura em um jogo para apoiar a aprendizagem de programação em vários níveis cognitivos." Anais do Simpósio Brasileiro de Informática na Educação. Vol. 24. No. 1. 
de Souza Pio, J. L., de Castro, T. H. C., and de Castro Júnior, A. N. (2006). "A robótica móvel como instrumento de apoio à aprendizagem da computação.” XVII Simpósio Brasileiro de Informática na Educação.

Fessakis, G., Evangelia Gouli, and E. Mavroudi. (2013) "Problem solving by 5-6 years old kindergarten children in a computer programming environment: A case study."Computers \& Education 63: 87-97.

Friedrich, Ronaldo Vaz, et al. (2012) "Proposta Metodológica para a Inserção ao Ensino de Lógica de Programação com Logo e Lego Mindstorms." Anais do Simpósio Brasileiro de Informática na Educação. Vol. 23. No. 1.

G Morati Jr, Roberto, et al. (2012) "Alice no Labirinto das Decisões: um jogo para exercitar a tomada de decisão e planejamento." Anais dos Workshops do Congresso Brasileiro de Informática na Educação. Vol. 1. No. 1.

Hulsey, Caitlin, Toni B. Pence, and Larry F. Hodges. (2014) "Camp CyberGirls: using a virtual world to introduce computing concepts to middle school girls." Proceedings of the 45th ACM technical symposium on Computer science education. ACM.

Melo, Leandro de Almeida, Thaíse Kelly de Lima Costa, and Any Caroliny Duarte Batista. (2013) "Pense bem: proposta e desenvolvimento de jogo digital para ensino de computação na educação básica." Anais do Simpósio Brasileiro de Informática na Educação. Vol. 24. No. 1.

Pietruchinski, Mônica Hoeldtke, et al. (2011) "Os jogos educativos no contexto do SBIE: uma revisão sistemática de Literatura." Anais do Simpósio Brasileiro de Informática na Educação. Vol. 1. No. 1.

Resnick, Mitchel, et al. (2009) "Scratch: programming for all." Communications of the ACM 52.11. (60-67).

Ribeiro, Paula Ceccon, Carlos Bazílio Martins, and Flávia Cristina Bernardini. (2011) "A Robótica como Ferramenta de Apoio ao Ensino de Disciplinas de Programação em Cursos de Computação e Engenharia." Anais do Workshop de Informática na Escola. Vol. 1. No. 1.

Salcedo, Sebastian Londoño, and Ana Maria Orozco Idrobo. (2011) "New tools and methodologies for programming languages learning using the scribbler robot and Alice." Frontiers in Education Conference (FIE). IEEE.

Sales, Chrystian Gesteira, and Vanessa Farias Dantas. (2010) "ProGame: um jogo para o ensino de algoritmos e programação." Anais do Simpósio Brasileiro de Informática na Educação. Vol. 1. No. 1.

The Csta Standards Task Force. CSTA K-12 Computer Science Standards. (2011). New York: ACM Computer Science Teachers Association. Disponível em: <http://csta.acm.org/Curriculum/sub/K12Standards.html>. Acesso em: 1 jul. 2014.

Vahldick, A., Benitti, F. B. V., Urban, D. L., Krueger, M. L., \& Halma, A. (2009). "O uso do Lego Mindstorms no apoio ao Ensino de Programação de Computadores." Anais do XX WEI Workshop de Educação Em Computação, Bento Gonçalves. (pp. 523-526).

Wing, J. M. (2006) Computational thinking. Communications of the ACM, v. 49, n. 3, p. 33-35.

Zilli, Silvana do Rocio. (2004) "A Robótica Educacional no Ensino Fundamental: perspectivas e prática.” Dissertação de Mestrado. Programa de Pós-Graduação em Engenharia de Produção. Universidade Federal de Santa Catarina. 